\title{
Fibrosing alveolitis in patients with rheumatoid arthritis as assessed by high resolution computed tomography, chest radiography, and pulmonary function tests
}

\author{
J K Dawson, H E Fewins, J Desmond, M P Lynch, D R Graham
}

Department of Rheumatology, St Helens and Knowsley Trust Hospitals, Liverpool, UK J K Dawson M P Lynch

\section{Department of} Radiology, St Helens and Knowsley Trust Hospitals

J Desmond

Department of Respiratory Medicine, St Helens and Knowsley Trust Hospitals

D R Graham

Department of Radiology, Cardiothoracic Centre, Liverpool, UK H E Fewins

Correspondence to: Dr J K Dawson, Department of Rheumatology, Southport and Formby District General Hospital, Southport, Merseyside PR8 6PN, UK twodocs@doctors.org.uk

Received 20 September 2000 Returned to authors 1 March 2001 Revised version received 2 April 2001 Accepted for publication 17 May 2001

\begin{abstract}
Background-Fibrosing alveolitis (FA) is a common and serious complication of rheumatoid arthritis (RA). Before the availability of high resolution computed tomographic (HRCT) scanning, it was difficult to diagnose accurately without recourse to biopsy. Prospective studies have reported a prevalence of interstitial lung disease (ILD) of 19-44\%. The term ILD used by these authors encompasses a variety of appearances on HRCT scans. This prospective study used HRCT scanning to determine the true prevalence of FA in hospital outpatients with RA, and to study associated clinical characteristics.

Methods-One hundred and fifty consecutive patients with RA were selected from a hospital outpatient department, irrespective of the presence or absence of chest disease. All underwent a detailed clinical assessment, chest HRCT scanning, and conventional chest radiography within 4 weeks of full pulmonary function tests. Results-Seventy percent of patients were current or reformed cigarette smokers. Twenty eight (19\%) had FA, most frequently of reticular pattern, and 12 of this group (43\%) also had emphysematous bullae. None of the previously suggested risk factors for developing FA were confirmed. Fifty four percent of patients with HRCT evidence of FA had bilateral basal chest crackles, $82 \%$ had a reduced carbon monoxide transfer factor (TLCO), $14 \%$ had restrictive pulmonary function tests, and $14 \%$ had bilateral chest radiographic signs of FA.
\end{abstract}

Conclusions-HRCT evidence of FA was present in $19 \%$ of hospital outpatients with RA. Abnormalities on chest examination or on full pulmonary function tests, even without restrictive changes or chest radiographic abnormalities, should prompt physicians to request a chest HRCT scan when investigating dyspnoea in patients with RA.

(Thorax 2001;56:622-627)

Keywords: fibrosing alveolitis; rheumatoid arthritis; high resolution computed tomography

Rheumatoid arthritis (RA) is a common disease that afflicts approximately $1 \%$ of the population, and perhaps up to $5 \%$ of women over the age of 65 years. Ellman and Ball first noted the association between fibrosing alveolitis (FA) and RA in $1948 .{ }^{1}$ It is now known that this is both the most common and most serious pleuropulmonary complication of RA. ${ }^{2}$ Although the course of FA is highly variable, the median survival of patients with diffuse interstitial fibrosis treated in hospital is less than 4 years. $^{3}$

The introduction of HRCT scanning since the late 1980s has revolutionised the approach to FA. The pattern of abnormality may be characteristic in certain diseases and virtually pathognomonic in most cases of cryptogenic fibrosing alveolitis (CFA). ${ }^{4}$ Studies have shown that chest HRCT scanning is more sensitive than plain chest radiography and more accurate in diagnosing $\mathrm{FA} .^{5}$

The literature on FA in patients with RA is confusing and varies from series to series, depending on diagnostic criteria. Chest radiographic changes occur in $1-5 \%$ of patients with RA, depression of carbon monoxide transfer factor (TLCO) in up to $41 \%,{ }^{6}$ and pulmonary fibrosis in $60 \%$ of volunteers undergoing open lung biopsy. ${ }^{7}$ HRCT based studies have reported changes consistent with FA in up to $62.6 \%$ of patients with RA. However, these studies have been retrospective in symptomatic patients $^{8-10}$ or the appearances consistent with interstitial lung disease (ILD) have not been specifically identified as being in keeping with FA. ${ }^{11} 12$

The aim of this study was to determine the prevalence of FA, as assessed by HRCT scanning, in a hospital outpatient population of patients with RA. By investigating a large cohort of patients with RA with a systematic prospective protocol, including clinical features and lung function together with HRCT scanning, we aimed to identify the clinical characteristics of patients with FA.

\section{Methods}

SUBJECTS

The study population consisted of 150 consecutive patients attending St Helens and Knowsley Trust Hospitals rheumatology outpatient departments. This hospital is based in North West England and its catchment population is from an industrialised area. Patients were diagnosed with definite RA, as defined by the American Rheumatism Association 1987 revised criteria, ${ }^{13}$ and enrolled irrespective of 
Table 1 Patient characteristics

\begin{tabular}{ll}
\hline Total number & 150 \\
F:M & $100: 50$ \\
Mean (SD) age (years) & $58.9(10.3)$ \\
Mean (SD) duration of RA (years) & $12.7(8)$ \\
Mean (SD) HAQ score & $1.7(0.7)$ \\
Rheumatoid factor positive & $116(77 \%)$ \\
Smokers & $58(39 \%)$ \\
Ex-smokers & $47(31 \%)$ \\
Non-smokers & $45(30 \%)$ \\
On methotrexate & $66(44 \%)$ \\
\hline SD = standard deviation; HAQ = Health Assessment Question- \\
naire (functional score, range 0-3).
\end{tabular}

chest symptoms or signs. Patients were excluded if they were pregnant, planning a pregnancy, or were known to have extrinsic allergic alveolitis, asbestosis, pneumoconiosis, or Caplan's syndrome.

The study was approved by the research ethics committee of St Helens and Knowsley Trust Hospitals.

CLINICAL ASSESSMENT

A questionnaire was completed on each patient by JKD. This noted duration of RA, extraarticular complications, current and previous disease modifying drugs, corticosteroid use, early morning joint stiffness, and patient assessment of disease activity. Each patient filled in the Modified Standford Health Assessment Questionnaire to assess functional impairment and so provide a measure of long term disease severity. Secondary sicca syndrome was tested using Schirmer's test for 5 minutes on each eye with less than $5 \mathrm{~mm}$ of wetting being considered a positive result. Respiratory questions were asked relating to previous chest disease, cough, dyspnoea, sputum production, chest pain, weight loss, and risk factors for respiratory disease such as smoking, medications, domestic pets, and occupation. Cigarette consumption was evaluated in pack years (one pack year $=20 /$ day for 1 year). Current smokers were those who had smoked during the previous 6 months; non-smokers had smoked less than 20 packets of cigarettes during their lifetime A detailed clinical examination was performed.

Table 1 shows the patient details. All patients had venous blood taken for measurement of full blood count, plasma viscosity, renal and liver function, $\mathrm{C}$ reactive protein, and plasma proteins. Immunological investigations included immunoglobulins, rheumatoid factor (latex agglutination, positive at $1 / 20$ dilution), and antinuclear antibody. All patients underwent echocardiography, electrocardiography (ECG), chest radiography, HRCT scanning, and full pulmonary function tests.

PULMONARY FUNCTION TESTING

Lung function was measured using a standard protocol and included spirometric tests (Sensor Medics water spirometer, California, USA), lung volumes (Sensor Medics helium dilution analyser), and carbon monoxide transfer factor (TLCO) (Sensor Medics autolink breath system). The highest of three reproducible measurements was used and expressed as $\%$ predicted for age, height, and sex according
Table 2 Abnormalities detected on thoracic HRCT scan

\begin{tabular}{lc}
\hline Abnormalities & Total \\
\hline Fibrosing alveolitis & 28 \\
$\quad$ predominantly reticular pattern & 22 \\
predominantly ground glass & 3 \\
ground glass = reticular pattern & 3 \\
Thickened interlobular lines & 28 \\
Bronchiectasis typical of obstructive lung disease & 12 \\
Bronchiolectasis & 2 \\
Emphysematous bullae & 23 \\
Decreased attenuation of pulmonary parenchyma & 5 \\
$\quad$ suggestive of air trapping & 1 \\
Mosaic perfusion pattern & 12 \\
Apical emphysematous bullae and FA & 7 \\
Pulmonary nodules & 5 \\
Pleural abnormalities & \\
\hline
\end{tabular}

to standardised tables. ${ }^{14}$ They were performed in the cardiorespiratory department at Whiston Hospital by one senior technician within 4 weeks of the HRCT scan.

\section{HRCT SCANNING}

All the study population underwent chest HRCT scanning using a Siemens Somatom hiQ scanner. Scanning time was $1.3 \mathrm{~s}$. Supine and prone views were taken. Serial slices $2 \mathrm{~mm}$ in width and $10 \mathrm{~mm}$ apart were taken. All images were obtained at window levels appropriate for lung parenchyma settings (window width $1300 \mathrm{HU}$; window level $-600 \mathrm{HU}$ ) and mediastinum (window width $350 \mathrm{HU}$; window level $40 \mathrm{HU}$ ). A chest radiograph was taken at the same time as the HRCT scan. The HRCT scans were interpreted and graded for FA by two consultant radiologists who were blind to the clinical details. Each radiologist reviewed the scans independently of the other and a consensus opinion between them was taken in the event of disagreement.

\section{STATISTICAL ANALYSIS}

The Mann-Whitney $U$ test was used to compare quantitative data and $\chi^{2}$ with Yates' correction was used to compare frequencies. The Student's $t$ test was used to compare normally distributed quantitative data. Interobserver variability before consensus agreement was evaluated for the presence of FA on HRCT pattern. Agreement between observers was expressed as a kappa value, with values of $0.40-0.75$ taken to indicate fair to good agreement between observers. Potentially significant parameters were tested for possible interrelationship by multiple logistic regression analysis. All statistical analyses were performed with version 9 SPSS software package (SPSS, Chicago, IL, USA).

INTERPRETATION OF HRCT SCAN

A ground glass pattern was defined as a patchy or diffuse increase in lung density that did not obscure pulmonary vasculature. A reticular pattern was defined as the presence of intersecting lines that formed anything from a fine network to frank honeycombing, ${ }^{15}$ and was defined as FA if the appearances were thought to be typical of usual interstitial pneumonia. ${ }^{16}$ Other lung disease present on the HRCT scan was systematically noted. The kappa score for level of agreement between the radiologists on the presence of FA was 0.741 . 
Table 3 Clinical features of patients with rheumatoid arthritis with and without fibrosing alveolitis (FA)

\begin{tabular}{lccl}
\hline Clinical feature & $F A(n=28)$ & No FA $(n=122)$ & $\begin{array}{l}p \text { Value }\left(\chi^{2}\right. \\
\text { test })\end{array}$ \\
\hline Male & 9 & 41 & 1 \\
Subcutaneous nodules & 18 & 65 & 0.4 \\
Dyspnoea: NYHA grade II & 15 & 69 & 0.84 \\
Dyspnoea: NYHA grade III & 5 & 8 & 0.07 \\
Productive cough & 13 & 44 & 0.39 \\
Never smoked & 5 & 40 & 0.17 \\
Finger clubbing & 5 & 11 & 0.182 \\
Bibasal crackles & 15 & 11 & $<0.001$ \\
CXR diagnostic of FA & 4 & 6 & 0.09 \\
Restrictive PFTs & 4 & 5 & 0.06 \\
Rheumatoid factor positive & 25 & 91 &
\end{tabular}

NYHA $=$ New York Health Association grading of dyspnoea; $\mathrm{CXR}=$ chest radiograph; $\mathrm{PFT}=$ pulmonary function test.

Table 4 Clinical features of patients with rheumatoid arthritis ( $R A$ ) with and without fibrosing alveolitis (FA)

\begin{tabular}{llll}
\hline Clinical feature & FA & No FA & p Value \\
\hline Age (years) & $61.2(8.7)$ & $58.3(10.6)$ & 0.37 \\
Duration of RA (years) & $11(6.4)$ & $13(8.2)$ & 0.374 \\
HAQ score & $1.8(0.76)$ & $1.6(1.9)$ & 0.183 \\
Cigarette (pack years) & $22.8(17.7)$ & $17.2(19)$ & 0.071 \\
Haemoglobin(g/dl) & $13.1(1.37)$ & $13.2(1.35)$ & 0.604 \\
C reactive protein(mg/l) & $34.3(31.7)$ & $28.5(29.3)$ & 0.47 \\
\hline
\end{tabular}

Values are mean (SD).

HAQ = Health Assessment Questionnaire (range 0-3).

Table 5 Pulmonary function tests in patients with rheumatoid arthritis (RA) with and without fibrosing alveolitis (FA)

\begin{tabular}{llll}
\hline Characteristic & $F A(n=28)$ & No $F A(n=122)$ & p Value \\
\hline${\text { Mean (SD) } \mathrm{FEV}_{1}(\mathrm{l})}_{\text {Mean (SD) } \mathrm{FEV}_{1} / \mathrm{FVC}(\%)}$ & $2.2(0.7)$ & $2.2(0.79)$ & $0.712^{\star}$ \\
Mean (SD)TLO & $59.8(10.41)$ & $72.3(10.2)$ & $0.024^{\star}$ \\
Mean (SD) \% predicted TLCO & $63(20.05)$ & $74.3(20.34)$ & $<0.001^{\star}$ \\
$\quad$ corrected for haemoglobin & & $77.6(21.48)$ & $0.001^{\star}$ \\
Percentage FVC <80\% predicted (n) & 7 & 23 & $0.64 \dagger$ \\
TLCo <75\% predicted (n) & 23 & 63 & $0.006 \dagger$ \\
RV < $<0 \%$ predicted (n) & 10 & 33 & $0.49 \dagger$ \\
VC $<80 \%$ predicted (n) & 8 & 18 & $0.14 \dagger$
\end{tabular}

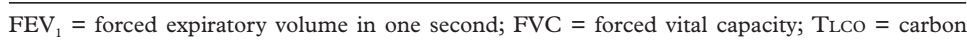
monoxide transfer factor; $\mathrm{RV}=$ residual volume; $\mathrm{VC}=$ vital capacity.

*Mann-Whitney U test.

$+\chi^{2}$ test.

\section{Results}

HRCT FINDINGS

HRCT findings are shown in table 2. HRCT diagnosed FA was found in 28 patients (19\%), only three of whom had chest signs, restrictive pulmonary function tests, and chest radiographic appearances typical of FA. Fifteen patients had bibasal crackles, all of whom had a predominantly reticular or reticular and ground glass pattern FA on the HRCT scan.

Twelve patients had bronchiectasis which was clinically evident in five. Where both FA and bronchiectasis were present in the same patient, this occurred at different areas of the lung and radiological features were suggestive of airway disease rather than traction bronchiectasis.

Apical emphysematous bullae were visible in 12 of the 28 patients (43\%) with FA, all of whom were current or reformed cigarette smokers.

CLINICAL FEATURES

Clinical features of the patients, with and without HRCT evidence of FA, are compared in tables 3 and 4 . The only clinical feature found to be significantly associated with the presence of FA was the presence of bibasal crackles $(\mathrm{p}<0.001)$. Finger clubbing was found in only five patients with FA.

Drug treatment including prednisolone and all disease modifying drugs was analysed but no significant association with FA was found in any group. No difference was identified between those with and without HRCT evidence of FA when the laboratory investigations were compared.

\section{PULMONARY FUNCTION TESTS}

The results of pulmonary function tests are shown in table 5. A statistically significantly difference was seen between the groups in the ratio of forced expiratory volume in one second to forced vital capacity $\left(\mathrm{FEV}_{1} / \mathrm{FVC}\right)$ and in TLCO and TLCO corrected for haemoglobin. Clinically, when pulmonary function tests with a restrictive pattern and lung volume readings below the normal range $(<80 \%$ predicted) were considered, there was no significant difference between the two groups with only a TLCO below the normal range $(<75 \%$ predicted) remaining significantly different.

Pulmonary function tests in patients with RA revealed that $52 \%$ of patients had a reduced TLCO without having FA. Even after correction for anaemia, this is still reduced in 55 of the patients without FA (45\%). In 29 patients the pulmonary function tests and HRCT scan suggested that the reduction in TLCO was a result of obstructive lung disease. In four others physical incapacity resulted in their inability to achieve a vital capacity above $80 \%$ predicted and, in such situations, TLCO is less reliable. Fourteen patients had thickened interlobular lines or pleural abnormalities that may have been the cause of the reduced TLCO, leaving eight patients with an unexplained isolated reduction in TLCO.

\section{CHEST RADIOGRAPHY}

As reported by other groups, chest radiography was insensitive in diagnosing FA and was not independently predictive of FA. Only four patients had chest radiographic changes consistent with FA, a further five with FA had unilateral fibrotic radiographic shadowing (four basal), one patient had basal consolidation, leaving 18 ( $64 \%$ of those with FA) with a normal chest radiograph or changes consistent with obstructive airways disease.

\section{PREDICTION OF FA}

Multiple logistic regression analysis was performed to determine the characteristics that predicted FA. The presence of FA on the HRCT scan as judged by two consultant radiologists with a special interest in thoracic HRCT scanning was taken as the dependent variable. No risk factors were found in patients with RA that predicted the occurrence of FA. As found in univariate analysis, the presence of bibasal crackles $(\mathrm{p}<0.0001), \% \mathrm{FEV}_{1} / \mathrm{FVC}$ $(p=0.03)$, and TLCO $(p=0.009) \quad$ (estimated odds ratio $0.0875,95 \%$ confidence interval 0.0332 to 0.2301 ) were significantly associated with FA on the HRCT scan. This logistic equation identified 25 out of 28 cases of FA. 
Table 6 Predictors of fibrosing alveolitis (FA) on HRCT scan

\begin{tabular}{lccll}
\hline Feature & $F A(n=28)$ & $\begin{array}{l}\text { No } F A \\
(n=122)\end{array}$ & Sensitivity & $\begin{array}{l}\text { Positive predictive } \\
\text { value }\end{array}$ \\
\hline NYHA grade III & 5 & 8 & $18 \%$ & $38 \%$ \\
Bibasal crackles & 15 & 11 & $54 \%$ & $58 \%$ \\
TLCo $<75 \%$ predicted & 23 & 63 & $82 \%$ & $27 \%$ \\
RV <80\% predicted & 10 & 33 & $36 \%$ & $23 \%$ \\
Restrictive PFT & 4 & 5 & $14 \%$ & $44 \%$ \\
CXR-FA & 4 & 6 & $14 \%$ & $40 \%$
\end{tabular}

NYHA $=$ New York Health Association grading for dyspnoea; TLCO = carbon monoxide transfer factor; $\mathrm{RV}=$ residual volume; $\mathrm{PFT}=$ pulmonary function test $\mathrm{CXR}=$ chest radiograph .

Table 6 shows abnormal findings in terms of sensitivity and positive predictive values. A reduced transfer factor is the single most sensitive test for FA and the presence of bibasal crackles is the clinical feature that gives the best positive predictive value.

\section{Discussion}

This study was designed to assess the prevalence of FA as this has a significant morbidity and mortality in patients with RA. Chest HRCT scanning has been shown to correlate with lung biopsy findings in FA in up to $93 \%$ of cases. ${ }^{4}$ Patients have been taken from a typical district general hospital outpatients department, irrespective of the presence or absence of lung disease. We have prospectively assessed the lungs of 150 patients with RA clinically, physiologically, by chest radiography, and thoracic HRCT scanning.

In this large cohort of patients with RA we have diagnosed FA in $19 \%$ by HRCT scanning. In $82 \%$ of these patients the RA associated FA was predominantly of a reticular pattern. In our patients $43 \%$ of those with FA also had apical emphysematous bullae visible on the HRCT scan. This association occurred in patients who were current smokers or in those who had previously smoked. We did not find emphysematous changes in addition to FA of a pure ground glass pattern. The association between emphysema and CFA has previously been described. ${ }^{17}$ It is thought to be coincidental and related to the patients' smoking habits as an association with alpha-1-antitrypsin has not been consistently found. ${ }^{18}$

Our observation that purely restrictive pulmonary function test results were not statistically significant is interesting. This may be partly explained by the fact that HRCT scanning detects FA at a much earlier stage, before significant loss of lung volume. A further explanation is the relationship of obstructive lung disease with the patients' smoking habits. We found emphysema with preservation of lung volumes in nine of the 12 patients with dual lung disease.

In our population a reduced TLCO was the most sensitive test for predicting the presence of FA on the HRCT scan, with eight patients having an unexplained isolated reduction in TLCo. There are two possible explanations for this finding. Firstly, and as suggested by other researchers, other systemic aspects of RA such as vascular disease ${ }^{19}$ or inflammatory activity ${ }^{20}$ may influence TLCO. Tumour necrosis factor $\alpha$ $(\mathrm{TNF} \alpha)$ is an important mediator of inflammation in $\mathrm{RA}^{21}$ and has been shown to alter alveolar permeability in other situations where
TNF $\alpha$ is released..$^{22}$ Secondly, TLco may be more sensitive than HRCT scanning in identifying FA in some patients. The $96 \%$ limits of normality for percentage predicted TLCO are $75 \%$ to $125 \%$, ${ }^{23}$ which means that a patient with a TLCO of $70 \%$ at presentation could have lost between $5 \%$ and $55 \%$ of their gas transfer. In the situation where the developing lung disease has caused a fall in gas transfer of $5 \%$ from the previous normal level for that patient, it may be that FA is not yet visible on the HRCT scan although the TLCO is below the normal range. This would be in keeping with reports of milder forms of idiopathic pulmonary fibrosis that are not evident on HRCT scans but have been found in lung biopsy specimens. ${ }^{24} \mathrm{We}$ intend to follow this cohort of patients for 3 years to determine if further signs of FA develop.

Previous HRCT based studies are of limited value because they used retrospective review of case notes to determine the clinical state and no formal comparison with pulmonary function tests was made. Four prospective studies have reported a prevalence of ILD of 19$44 \% .^{11}{ }^{12} 2526$ One reason for the variability in the prevalence of ILD is the terminology used by different authors. The HRCT appearance in keeping with "ILD" encompasses a variety of abnormalities including bronchiectasis, ${ }^{11}$ interstitial fibrosis, ${ }^{12}$ thickening of non-septal and septal lines, ${ }^{11}$ ground glass attenuation, ${ }^{11}{ }^{25-27}$ honeycombing, ${ }^{11}{ }^{25-27}$ and traction bronchiectasis. ${ }^{11}$ The HRCT study by Fewins et $a l^{11}$ of patients with RA revealed a high prevalence of ILD $(44 \%)$. The descriptions of the ILD patterns were not sufficiently detailed to determine the exact frequency of FA type ILD. If all forms of our HRCT diagnosed ILD - that is, FA and interlobular thickening-are combined, there is a closer level of prevalence (37\%) of ILD to that study.

McDonagh and colleagues studied a population with RA from the North East of England, $80 \%$ of whom were smokers, ${ }^{25}$ and found a $20 \%$ prevalence of FA when the 20 patients in their RA case-control group, thought to be without lung disease, were investigated by HRCT scanning. When the clinical features of all the patients with RA and FA were analysed, they also found a low incidence of finger clubbing. The frequent association of emphysema and FA was also noted. When the pulmonary function of all the patients with FA was compared with that of patients with a normal HRCT scan or nodules or pleural disease alone, reductions in $\mathrm{FEV}_{1}$ and TLCo became significant in this group. Our patients did not have a significant reduction in $\mathrm{FEV}_{1}$, which may be because there were more smokers in the study by McDonagh et al. Cortet et $a l^{26}$ prospectively assessed HRCT scans and pulmonary function tests in patients with RA of whom only $23.5 \%$ were smokers; $30 \%$ were found to have bronchiectasis and this was the focus of their paper. Ground glass changes were observed on the HRCT scan in 11 patients $(17 \%)$ and honeycombing was seen in two $(2.9 \%)$. This is a similar prevalence to that found in our study, although the proportion 
with ground glass changes is quite different. In contrast to our findings and those of McDonagh et al, they found that ground glass attenuation was associated with a significant decrease in FVC but not with a low TLCo. These differences may be partly explained by the different smoking habits of the study groups resulting in less frequent dual lung disease in the patients studied by Cortet et al.

We did not find any relation between the presence of FA and previously described predisposing factors such as male sex, nodular disease, extra-articular disease, longer disease duration, or disease severity. This lack of association has also been confirmed in one other HRCT centred study ${ }^{8}$ where these clinical features have been recorded. Gabbay et al studied 36 patients with RA of less than 2 years' duration prospectively for ILD. ${ }^{11}$ The analysis for risk factors for developing clinically significant ILD was ultimately derived from only five patients with this complication. They noted an association between male sex and clinically significant lung disease in their study of patients with early onset RA, a different population from ours.

Saag et $a l^{28}$ recently reported that smoking was the most consistent independent risk factor predicting the development of ILD in RA. Notably, the diagnosis of ILD was based on reduced FVC, TLCO $(<80 \%)$ and/or chest radiographic interstitial infiltrates. We and others have shown that the sensitivity and specificity of these investigations are inferior to HRCT scanning in diagnosing FA and therefore the results should be interpreted with caution. We assessed cumulative cigarette exposure and found that this had the strongest association with the development of FA. However, in contrast to Saag et al, it did not reach statistical significance.

A $19 \%$ prevalence of FA in a cross section of a hospital outpatient population with RA that can be detected non-invasively from an early stage by HRCT scanning may tempt physicians to consider routine HRCT scanning of all patients with RA. However, there is very little published work describing the course of outpatient diagnosed FA in patients with RA, and the high mortality figures are from patients studied after being admitted to hospital for their lung disease $^{3}$ or from tertiary referral centres. ${ }^{29}$ It would seem from clinical experience that many patients with RA have mild or subclinical forms of FA. ${ }^{30}$ Further work needs to be carried out to define which features of patients with HRCT diagnosed FA identify clinically significant disease before routine HRCT scanning of patients with RA is undertaken. Currently, we would recommend that, when investigating dyspnoea in patients with RA, chest radiography and full pulmonary function tests should be requested in addition to clinical examination. The presence of one or more of bibasal crackles, reduced $\mathrm{T}_{\mathrm{LCO}}$, or raised $\mathrm{FEV}_{1} / \mathrm{FVC}$ ratio would then be an indication for HRCT scanning. The association of emphysema with FA in a population of patients with RA who have a considerable smoking habit should be noted. Thirty two percent of our patients had obstructive pulmonary function tests or chest radiographic changes suggestive of chronic obstructive pulmonary disease (COPD) were found also to have FA on the HRCT scan. A normal chest radiograph or basic investigations suggesting a diagnosis of obstructive lung disease should not therefore deter a physician from investigating for additional FA by HRCT scanning.

Further work should now be directed towards defining the natural progression of HRCT diagnosed FA in patients with RA, and comparing the different HRCT patterns seen in RA with the extended classification of lung histology found in CFA. As yet, neither of these studies has been specifically performed on a population with RA, and this is essential before the development of well designed treatment trials.

At the time the study was devised, John Kenny was integral to the undertaking of the study as well as initial interpretation of chest radiographs and HRCT scans. It is with much regret that he had to retire due to ill health. All his work was greatly appreciated. We thank the staff at Whiston Hospital Cardiorespiratory Department, particularly Karen Eyres. We also thank Dr Rob Moots for providing his helpful suggestions and comments on the paper.

Funding: Financial support for the research was provided by the British Lung Foundation.

1 Ellman P, Ball RE. Rheumatoid disease with joint and pulmonary manifestations. BMF 1948;2:816-20.

2 Roschmann RA, Rothenberg R J. Pulmonary fibrosis in RA. A review of clinical features and therapy. Semin Arthritis Rheum 1987;16:174-85.

3 Hakala M. Poor prognosis in patients with rheumatoid arthritis hospitalised for interstitial lung fibrosis. Chest arthritis hospitalis

4 Mathieson JR, Mayo JR, Staples CA, et al. Chronic diffuse infiltrative lung disease: comparison of diagnostic accuracy of CT and chest radiography. Radiology 1989;171: $111-6$.

5 Padley SPG, Hansell DM, Flower CDR, et al. Comparative accuracy of HRCT and chest radiography in the diagnosis
of chronic diffuse infiltrative lung disease. Clin Radiol 1991;44:222-6.

6 Frank ST, Weg JG, Harkleroad LE, et al. Pulmonary dysfunction in rheumatoid disease. Chest 1973;63:27-34.

7 Cervantes-Perez P, Toro-Perez AH, Rodriguez-Jurado P. Pulmonary involvement in rheumatoid arthritis. $7 A M A$ 1980;243:1715-9.

8 Fujii M, Adachi S, Shimizu T, et al. Interstitial lung disease in rheumatoid arthritis: assessment with high- resolution in rheumatoid arthritis: assessment with high- resolution

9 Okuda Y, Takasugi K, Imai A, et al. Clinical study of rheuOkuda Y, Takasugi K, Imai A, et al. Clinical study of rheu-
matoid interstitial lung disease evaluated by high resolution matoid interstitial lung disease eva

10 Sasaka K, Nakajima Y, Kase C, et al. Plain film and CT findings of pulmonary involvement in rheumatoid arthritis. Ryumachi 1998;38:705-16.

11 Fewins HE, McGowan I, Whitehouse GH, et al. High definition computed tomography in rheumatoid arthritis associated pulmonary disease. $\mathrm{Br} \mathcal{F}$ Rheumatol 1991;30: 214-6.

12 Gabbay E, Tarala R, Will R, et al. Interstitial lung disease in recent onset rheumatoid arthritis. Am F Respir Crit Care Med 1997;156(2 Pt 1):528-35.

13 Arnett FCS, Edworthy M, Bloch DA, et al. The American Rheumatism Association Revised Criteria for the Classification of Rheumatoid Arthritis. Arthritis Rheum 1988;32: 315-24.

14 Quanjer PH. Standardized lung function testing. 1983 Report of the Working Party Standardization on Lung Function Testing. European Community for Coal and Steel. Bull Europ Physiopathol Respir 1983;19(Suppl 15):182.

15 Wells AU, Rubens MB, du Bois RM, et al. Serial CT in fibrosing alveolitis: Prognostic significance of the initial pattern. Am f Roentgenol 1993;161:1159-65.

16 Katzenstein A-L, Myers JL. Idiopathic pulmonary fibrosis. Clinical relevance of pathological classification. Am f Respir Crit Care Med 1998;157:1301-15.

17 Doherty MJ, Pearson MG, O'Grady EA, et al. Cryptogenic fibrosing alveolitis with preserved lung volumes. Thorax 1997;52:998-1002.

18 Hubbard R, Baoka Y, Kalsheker N, et al. Alpha-1antitrypsin phenotypes in patients with cryptogenic fibrosing alveolitis:a case control study. Eur Respir 7 1997;10: 2881-3. 
19 Oxholm P, Madsen EB, Manthorpe R, et al. Pulmonary function in patients with rheumatoid arthritis. Scand $\mathcal{F}$ Rheumatol 1982;11:109-12.

20 Schernthaner G, Scherak O, Kolarz G, et al. Seropositive rheumatoid arthritis associated with decreased diffusion capacity of the lung. Ann Rheum Dis 1976;35:258-62.

21 Deleuran BW, Chu CQ, Field M, et al. Localization of tumour necrosis factor receptors in the synovial tissue and cartilage-pannus junction in patients with rheumatoid arthritis. Implications for local actions of tumour necrosis arthritis. Implications for local actions of tum

22 Stephens KE, Ishizaka A, Larrick JW, et al. Tumour necrosis factor causes increased pulmonary permeability and edema. Am Rev Respir Dis 1988;137:1364-70.

23 Laszlo G. Testing the mechanics of breathing. Pulmonary function a guide for clinicians. Cambridge: Cambridge University Press, 1994.

24 Orens JB, Kazerooni EA, Martinez FJ, et al. The sensitivity of high-resolution CT in detecting idiopathic pulmonary fibrosis proved by open lung biopsy. A prospective study. Chest 1995;108:109-15.
25 McDonagh J, Greaves M, Wright AR, et al. High resolution computed tomography of the lungs in patients with cheumatoid arthritis and interstitial lung disease. $\mathrm{Br} f$ Rheumatol 1994;33:118-22.

26 Cortet B, Perez T, Roux N, et al. Pulmonary function tests and high resolution computed tomography of the lungs in patients with rheumatoid arthritis. Ann Rheum Dis 1997;56:596-600

27 Muller-Leisse C, Bussmann A, Meyer O, et al. Pulmonary manifestations in rheumatoid arthritis: high-resolution computed tomography in correlation with the skeletal changes and the laboratory chemical changes. Rofo Fortschr Geb Rontgenstr Neuen Bildgeb Verfahr 1996;165:438-44.

28 Saag KG, Kolluri S, Koehnke RK, et al. Rheumatoid arthritis lung disease. Determinants of radiographic and physiologic abnormalities. Arthritis Rheum 1996:39:1711-9.

29 Turner-Warwick M, Burrows B, Johnson A. Cryptogenic fibrosing alveolitis: clinical features and their influence on survival. Thorax 1980;35:171-80.

30 Wells AU, Hansell DM, du Bois RM. Interstitial lung disease in the collagen vascular diseases. Semin Respir Med 1993;14:333-43.

\section{1st Asia Pacific Forum on Quality Improvement in Health Care}

\section{Three day conference}

\section{Wednesday 19 to Friday 21 September 2001 Sydney, Australia}

We are delighted to announce this forthcoming conference in Sydney. Authors are invited to submit papers (call for papers closes on Friday 6 April), and delegate enquiries are welcome. The themes of the Forum are:

- Improving patient safety

- Leadership for improvement

- Consumers driving change

- Building capacity for change: measurement, education and human resources

- The context: incentives and barriers for change

- Improving health systems

- The evidence and scientific basis for quality improvement.

Presented to you by the BMJ Publishing Group (London, UK) and Institute for Healthcare Improvement (Boston, USA), with the support of the the Commonwealth Department of Health and Aged Care (Australia), Safety and Quality Council (Australia), NSW Health (Australia), and Ministry of Health (New Zealand).

For more information contact: quality@bma.org.uk or fax +44 (0)20 73836869 